\title{
Sur l'élément de Clough et Tocher
}

Revue française d'automatique, informatique, recherche opérationnelle. Analyse numérique, tome 8, no 2 (1974), p. 19-27.

<http://www.numdam.org/item?id=M2AN_1974_8_2_19_0>

(C) AFCET, 1974, tous droits réservés.

L'accès aux archives de la revue "Revue française d'automatique, informatique, recherche opérationnelle. Analyse numérique » implique l'accord avec les conditions générales d'utilisation (http://www.numdam.org/legal. php). Toute utilisation commerciale ou impression systématique est constitutive d'une infraction pénale. Toute copie ou impression de ce fichier doit contenir la présente mention de copyright.

\section{Numdam}

Article numérisé dans le cadre du programme

Numérisation de documents anciens mathématiques

http://www.numdam.org/ 


\title{
SUR L'ELEMENT DE GLOUGH ET TOGHER
}

\author{
par P. G. Ciarlet $\left(^{(1)}\right.$
}

Résumé. - On étudie les propriétés d'unisolvence et d'interpolation de l'élément fini de Clough et Tocher, utilisé dans la résolution numérique des problèmes de plaques.

\section{INTRODUCTION}

Nous utilisons les notations suivantes :

$\left.u\right|_{A}=$ restriction de la fonction $u$ à l'ensemble $A$,

$P_{k}=$ espace vectoriel des polynômes de deux variables, de degré $\leqslant k$, $[a, b]=$ segment joignant les deux points $a$ et $b$,

$$
D p=\left(\frac{\partial p}{\partial x}, \frac{\partial p}{\partial y}\right) \in R^{2 i}
$$

$\langle.,\rangle=$. produit scalaire dans $R^{2}$,

$\AA=$ intérieur de l'ensemble $A$,

$$
|v|_{m, K}=\left(\int_{K|\alpha|=m}\left|\partial^{\alpha} v\right|^{2} \mathrm{~d} x \mathrm{~d} y\right)^{1 / 2} .
$$

L'objet de cet article est d'étudier l'élément fini de Clough et Tocher, introduit en [5]. Cet élément est utilisé dans la résolution numérique des problèmes de plaques; à ce sujet, voir par exemple [1], [2], [7, Chapitre 10].

L'élément fini est un triangle $K$, qui est lui-même subdivisé en trois triangles $K_{i}, 1 \leqslant i \leqslant 3$ (cf. fig. 1), le point $a=\bigcap_{i=1}^{3} K_{i}$ étant un point seulement supposé intérieur au triangle $K$.

(1) Analyse Numérique, Université de Paris VI.

Revue Française d'Automatique, Informatique et Recherche Opérationnelle no août 1974, R-2. 
On associe à l'élément $K$ un espace $P$ de fonctions réelles définies sur $K$, en posant

$$
P=\left\{p \in \mathcal{C}^{1}(K) ;\left.p\right|_{K_{i}} \in P_{3}, 1 \leqslant i \leqslant 3\right\}
$$

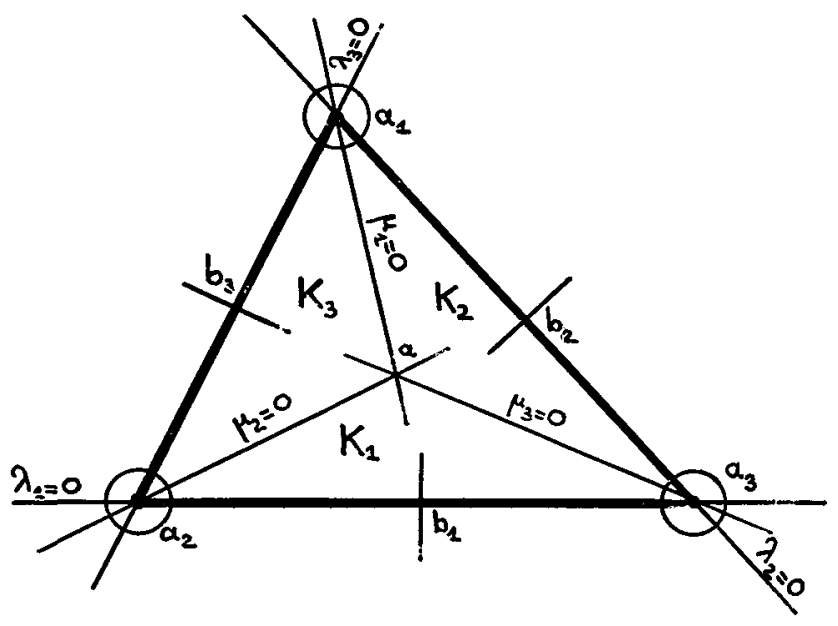

Figure 1

Si $p$ est une fonction quelconque de l'espace $P$, on lui associe l'ensemble des degrés de liberté défini par

$$
\begin{aligned}
\Sigma=\left\{p\left(a_{i}\right), 1 \leqslant i \leqslant 3 ; \frac{\partial p}{\partial x}\left(a_{i}\right),\right. & \\
1 & \left.\leqslant i \leqslant 3 ; \frac{\partial p}{\partial y}\left(a_{i}\right), 1 \leqslant i \leqslant 3 ; \frac{\partial p}{\partial n}\left(b_{i}\right), 1 \leqslant i \leqslant 3\right\},
\end{aligned}
$$

$\frac{\partial p}{\partial n}\left(b_{i}\right)$ étant la dérivée normale extérieure au milieu $b_{i}$ du côté $\left[a_{i+1}, a_{i+2}\right]$, les indices $i$ étant comptés modulo 3, ainsi que dans la suite de cet article.

La dimension de l'espace $P_{3}$ étant 10 , il faut 30 équations pour déterminer les trois fonctions

$$
p_{i}=\left.p\right|_{k_{i}}, 1 \leqslant i \leqslant 3 \text {. }
$$

Il est facile de voir que la donnée de l'ensemble $\Sigma$ des degrés de liberté entraîne la donnée de 21 équations, les 9 équations restantes provenant de la condition $" p \in \mathcal{C}^{1}(K) »$, que l'on prend en compte en écrivant par exemple que la valeur des trois fonctions $p_{i}$ ainsi que de leurs dérivées partielles premières sont les mêmes au point $a$ (6 équations) et que les dérivées normales sont les mêmes aux milieux des côtés $\left[a, a_{i}\right]$ (3 équations). Ce décompte fait, 
il reste à montrer que la matrice du système linéaire obtenu est régulière, autrement dit que l'ensemble $\Sigma$ de (1.2) est " $P$-unisolvent », suivant la terminologie de [3]. C'est ce que nous faisons au Théorème 1.

Naturellement l'espace $P$ vérifie l'inclusion $P_{3} \subset P$, mais on ne peut pas appliquer directement la théorie générale de l'interpolation pour des familles affines, que nous appellerons dorénavant théorie affine, telle qu'elle a été développée en [3]. En effet, la théorie affine ne permet pas d'avoir un point $a$ variable, et par ailleurs, certains degrés de liberté sont des valeurs de dérivées normales, qui ne sont pas invariantes par transformation affine. Nous montrons au Théorème 2 comment la théorie affine peut cependant être adaptée pour tenir compte des deux difficultés ci-dessus.

\section{P-UNISOLVENCE DE L'ENSEMBLE $\Sigma$}

L'espace $P$ et l'ensemble $\Sigma$ sont définis comme en (1.1) et (1.2), respectivement.

Théorème 1. L'ensemble $\Sigma$ des degrés de liberté est P-unisolvent, i.e., les 12 valeurs $p\left(a_{i}\right), \frac{\partial p}{\partial x}\left(a_{i}\right), \frac{\partial p}{\partial y}\left(a_{i}\right), \frac{\partial p}{\partial n}\left(b_{i}\right), 1 \leqslant i \leqslant 3$, définissent une et une seule fonction $p$ de l'espace $P$.

Démonstration. La $P$-unisolvence équivalant à la résolution d'un système linéaire à matrice carrée, il suffit de montrer que si tous les degrés de liberté sont nuls, alors la fonction correspondante est nécessairement nulle. La démonstration va comporter plusieurs étapes.

(i) Préliminaires géométriques. On suppose que $a=(0,0)$. Soient $\left(x_{i}, y_{i}\right)$ les coordonnées des points $a_{i}, 1 \leqslant i \leqslant 3$; les déterminants

$$
\alpha_{i}=\operatorname{dét}\left[\begin{array}{ll}
x_{i+1} & y_{i+1} \\
x_{i+2} & y_{i+2}
\end{array}\right] \quad, \quad 1 \leqslant i \leqslant 3 .
$$

sont tous différents de 0 puisque le point $a$ n'appartient à aucun des côtés du triangle. D'autre part, comme les trois points $a_{i}$ définissent un véritable triangle si et seulement si la quantité

$$
\alpha=\operatorname{dét}\left[\begin{array}{lll}
x_{1} & x_{2} & x_{3} \\
y_{1} & y_{2} & y_{3} \\
1 & 1 & 1
\end{array}\right]=\sum_{i=1}^{3} \alpha_{i}
$$

est différente de zéro, on peut désormais supposer que

sans restreindre la généralité.

$$
\alpha=1,
$$

$n^{\circ}$ août 1974, R-2. 
L'équation $\lambda_{i}(x, y)=0$ du côté $\left[a_{i+1}, a_{i+2}\right]$ est déterminée par la condition $\lambda_{i}(0,0)=1$, de sorte que l'équation $\mu_{i}(x, y)=0$, avec $\mu_{i}=\lambda_{i+1}-\lambda_{i+2}$, est l'équation du côté $\left[a, a_{i}\right]$.

(ii) Considérons l'un des triangles $K_{i}$ (cf. fig. 2), par exemple le triangle $K_{1}$.

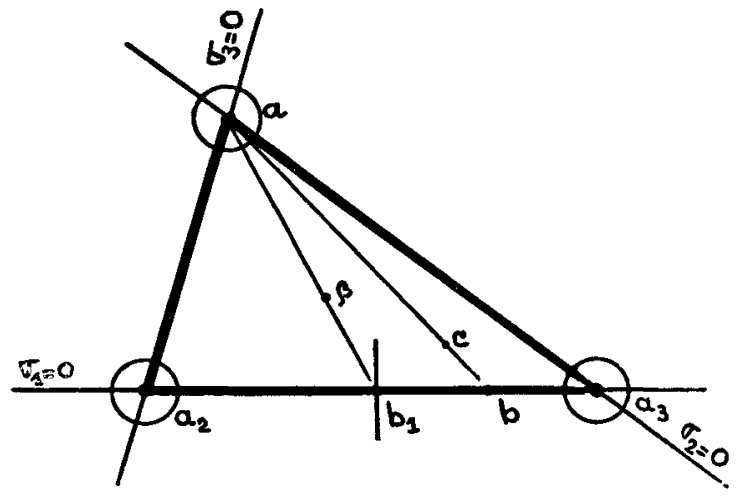

Figure 2

L'ensemble des degrés de liberté défini par

$$
\Sigma_{1}=\left\{p(.), \frac{\partial p}{\partial x}(.), \frac{\partial p}{\partial y}(.) \text { en } a, a_{2}, a_{3} ; \frac{\partial p}{\partial n}\left(b_{1}\right)\right\}
$$

est $P_{3}$-unisolvent. En effet, comme card $\left(\Sigma_{1}\right)=\operatorname{dim}\left(P_{3}\right)=10$, il suffit de montrer que si tous les degrés de liberté de $\Sigma_{1}$ sont nuls, le polynôme du troisième degré correspondant est identiquement nul. Soit $p$ un tel polynôme : alors le gradient $D p$ est nul le long du côté $\left[a_{2}, a_{3}\right]$. Soit ensuite $c$ un point quelconque du triangle $K_{1}$. Comme $p(a)=p(b)=0$ et $D p(a)=D p(b)=0$, il en résulte que $p(c)=0$.

En (iii), nous aurons besoin de l'expression d'un polynôme $p \in P_{3}$, nul ainsi que ses dérivées partielles premières le long du côté $\left[a_{2}, a_{3}\right]$. Elle est donnée par

$$
\begin{aligned}
p=\sigma_{1}^{2}\left\{-2 \sigma_{1}\right. & +3) p(a) \\
& \left.+\sigma_{2}\left\langle D p(a),\left(a_{2}-a\right)\right\rangle+\sigma_{3}\left\langle D p(a),\left(a_{3}-a\right)\right\rangle\right\},
\end{aligned}
$$

$s_{1}, \sigma_{2}$ et $\sigma_{3}$ étant respectivement les coordonnées barycentriques d'un point du triangle $K_{1}$ par rapport aux trois sommets $a, a_{2}$ et $a_{3}$, selon le numérotage de la figure 2.

Pour établir (2.1), on utilise les fonctions de base données dans [4, Théorème 5], et on remplace la valeur de la fonction $p$ au barycentre $\beta$ du triangle par l'expression $\left(7 p(a)+2\left\langle D p(a),\left(b_{1}-a\right)\right\rangle\right) / 27$, vérifiée par la fonction $p$, 
considérée le long du segment $\left[a, b_{1}\right]$ comme un polynôme d'une variable de degré $\leqslant 3$.

(iii) Considérons une fonction $p \in P$ dont tous les degrés de liberté sont nuls. D'après ce qui précède, il suffit de montrer que les quatre inconnues

$$
\delta_{0}=p(a) \text {, et } \delta_{i}=\alpha_{i}\left\langle D p(a),\left(a_{i}-a\right)\right\rangle, 1 \leqslant i \leqslant 3,
$$

sont nulles. Observons d'ailleurs que les trois dernières sont liées par la relation

$$
\sum_{i=1}^{3} \delta_{i}=0
$$

qui exprime la dérivabilité de la fonction $p$ au point $a$.

Considérons le triangle $K_{1}$, par exemple. Utilisant la relation (2.1) et les relations $\sigma_{1}=\lambda_{1}, \sigma_{2}=-\alpha_{2} \mu_{3}, \sigma_{3}=\alpha_{3} \mu_{2}$, on déduit que

$$
p_{1}=\lambda_{1}^{2}\left\{\left(-2 \lambda_{1}+3\right) \delta_{0}+\left(\lambda_{2}-\lambda_{1}\right) \delta_{2}+\left(\lambda_{3}-\lambda_{1}\right) \delta_{3}\right\},
$$

et naturellement, on obtient des expressions analogues à des permutations circulaires près, pour les fonctions $p_{2}$ et $p_{3}$.

Il nous reste à exprimer la dérivabilité de la fonction $p$ le long des segments $\left[a, a_{i}\right]$. Les fonctions $p_{i}$ pouvant être considérées comme définies dans tout le plan par l'intermédiaire des expressions (2.3), cela revient à montrer que les trois fonctions

$$
q_{i+2}=p_{i+1}-p_{i}, 1 \leqslant i \leqslant 3
$$

sont identiquement nulles, ainsi que leurs dérivées partielles premières, le long des segments $\left[a, a_{i+2}\right]$. Examinons par exemple la fonction $q_{3}=p_{2}-p_{1}$. Utilisant l'expression (2.2) et l'expression analogue pour $p_{2}$, ainsi que l'identité $\sum_{i=1}^{3} \alpha_{i} \lambda_{i}=1$, on obtient

$$
q_{3}=\left(\lambda_{2}-\lambda_{1}\right) \cdot\left(\begin{array}{c}
\left\{-2\left(\lambda_{1}^{2}+\lambda_{1} \lambda_{2}+\lambda_{2}^{2}\right)+3\left(\lambda_{1}+\lambda_{2}\right)\right\} \delta_{0} \\
-\lambda_{2}^{2} \delta_{1}-\lambda_{1}^{2} \delta_{2}-\left(\lambda_{1}^{2}+\lambda_{1} \lambda_{2}+\lambda_{2}^{2}\right) \delta_{3} \\
+\left(1-\alpha_{1} \lambda_{1}-\alpha_{2} \lambda_{2}\right)\left(\lambda_{1}+\lambda_{2}\right) \delta_{3} / \alpha_{3}
\end{array}\right),
$$

ce qui montre que la fonction $q_{3}$ est nulle le long du côté $\left[a, a_{3}\right]$. Comme $D q_{3}(a)=D q_{3}\left(a_{3}\right)=0$, il suffit d'exprimer par ailleurs que $D q_{3}\left(a_{3} / 2\right)=0$, par exemple. Compte tenu de l'expression (2.4), cette condition s'écrit

$$
\delta_{0}-\sum_{i=1}^{3} \delta_{i}+\frac{2}{\alpha_{j}} \delta_{j}=0
$$

avec $j=3$, et on obtiendrait des relations analogues avec $j=1$ et 2 . $\mathrm{n}^{\circ}$ août 1974, R-2. 
Dans ces conditions, les inconnues $\delta_{0}=p(a)$ et $\delta_{i}=\alpha_{i}\left\langle D p(a),\left(a_{i}-a\right)\right\rangle$, $1 \leqslant i \leqslant 3$, vérifient le système linéaire représenté par les équations (2.2) et (2.5). Le déterminant de la matrice de ce système linéaire valant $\frac{6}{\alpha_{1} \alpha_{2} \alpha_{3}}$, on en déduit que les inconnues sont toutes nulles, ce qui achève la démonstration.

\section{ERREUR D'INTERPOLATION}

A toute fonction $u$ suffisamment régulière définie sur l'élément $K$, par exemple partout dérivable dans $K$, on associe la fonction d'interpolation $\Pi u$ qui est par définition la (seule) fonction de l'espace $P$ dont les degrés de liberté sont les mêmes que ceux de la fonction $u$, i.e., $\Pi u\left(a_{1}\right)=u\left(a_{1}\right), \ldots, \frac{\partial \Pi u}{\partial n}\left(b_{3}\right)=\frac{\partial u}{\partial n}\left(b_{3}\right)$.

Soit $\hat{K}$ un triangle de référence et $\hat{B}$ un compact contenu dans $\stackrel{\hat{K}}{\mathrm{~K}}$, et soit par ailleurs $(K)$ une famille d'éléments du type décrit au $\S 2$; on pose

$$
h=\operatorname{diam}(K), \rho=\operatorname{diam}(\text { cercle inscrit dans } K) \text {. }
$$

On dit alors que la famille est régulière si les deux conditions qui suivent sont simultanément vérifiées.

(a) Il existe une constante $\alpha>0$ telle que $\alpha \leqslant \rho / h$ pour tous les éléments de la famille.

(b) Notons $F$ la (seule) transformation affine du plan qui vérifie $F\left(\hat{a}_{i}\right)=a_{i}, 1 \leqslant i \leqslant 3$ (cf. fig. 3). Alors le point $\hat{a}=\bar{F}^{1}(a)$ appartient au compact $\hat{B}$.
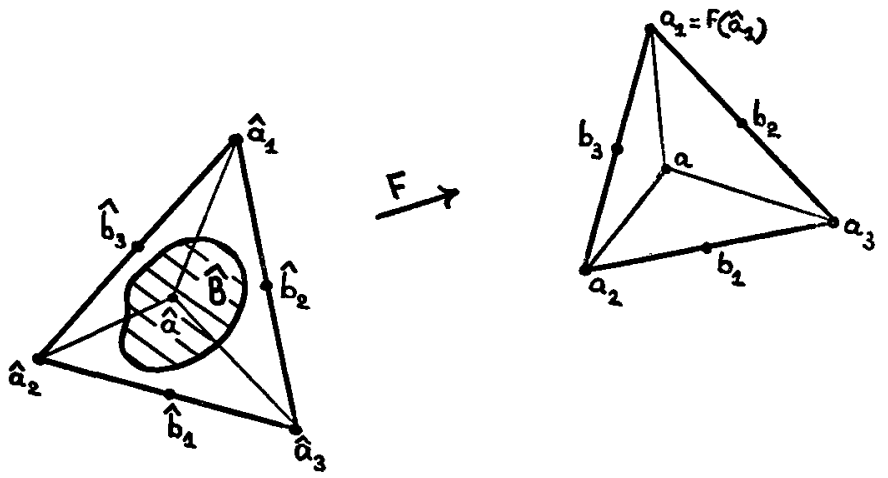

Figure 3

Remarquons que la condition (a) ci-dessus est la condition usuelle de régularité des familles affines d'éléments finis; cf. [3]. La condition supplémentaire (b) permet en somme de "faire varier » le point $a$ qui, autrement, serait 
assujetti à être l'image $F(\hat{a})$ d'un point $\hat{a} \in \hat{K}$ fixé une fois pour toutes. Naturellement cette condition devient superflue dans certains cas, par exemple si le point $a$ est toujours le barycentre du triangle $K$.

On a alors le

Théorème 2. Étant donné une famille régulière d'éléments de Clough et Tocher, il existe une constante $C$ indépendante des éléments de la famille telle que

$$
|u-\Pi u|_{m, K} \leqslant C h^{4-m}|u|_{4, K} \quad \text { pour } \quad m=0,1,2,
$$

pour toute fonction $u \in H^{4}(K)$.

Démonstration. On remarque pour commencer que, d'une part, la fonction d'interpolation $\Pi u$ est bien définie pour $u \in H^{4}(K)$ en vertu de l'inclusion $H^{4}(K) \subset \mathrm{C}^{2}(K)$, et que, d'autre part, on ne peut espérer avoir des majorations du type (3.1) pour des valeurs $m \geqslant 3$ puisqu'on a «seulement " l'inclusion $P \subset H^{2}(K)$.

$\mathrm{La}$ démonstration va comporter trois étapes.

(i) On suppose pour commencer qu'on considère une famille régulière pour laquelle le point â est le même pour tous les éléments de la famille. Étant donné un élément courant d'une telle famille, nous allons évaluer les seminormes $|u-\Lambda(\hat{a}) u|_{m, K}, m=0,1,2$, où $\Lambda(\hat{a}) u=\Lambda u$ est la (seule) fonction de l'espace $P$ qui vérifie

$$
\begin{gathered}
\Lambda u\left(a_{i}\right)=u\left(a_{i}\right), D \Lambda u\left(a_{i}\right)=D u\left(a_{i}\right), \quad 1 \leqslant i \leqslant 3, \\
\left\langle D \Lambda u\left(b_{i}\right),\left(a-b_{i}\right)\right\rangle=\left\langle D u\left(b_{i}\right),\left(a-b_{i}\right)\right\rangle, \quad 1 \leqslant i \leqslant 3,
\end{gathered}
$$

l'existence et l'unicité d'une telle fonction d'interpolation résultant encore de la démonstration du Théorème 1 .

De la sorte, la théorie affine peut être utilisée : compte tenu de l'inclusion fondamentale $P_{3} \subset P$, l'application du Théorème 5 de [3] à l'application $\Lambda(\hat{a})$, pour $m=0,1,2$, conduit à des majorations du type

$$
|u-\Lambda(\hat{a}) u|_{m, K} \leqslant C(\hat{a}, \hat{K}) h^{4-m}|u|_{4, K} \quad \text { pour } \quad m=0,1,2,
$$

pour une constante $C(\hat{a}, K)$ ne dépendant que du triangle de référence et du point $\hat{a}$.

(ii) Pour prendre en compte la possibilité d'avoir un point $\hat{a}$ "variable» dans le compact $\hat{B}$, on utilise le fait que (cf. Lemme 7 de [3]) :

$$
C(\hat{a}, \hat{K})=C(\hat{K})\|I-\hat{\Lambda}(\hat{a})\|_{\mathfrak{L}_{\left(H^{4}(\hat{K}) ; H^{m}(\hat{K})\right)}}
$$

où $C(\hat{K})$ est une constante ne dépendant que de $\hat{K}, I$ est l'identité et $\hat{\Lambda}(\hat{a})$ est l'application qui à toute fonction $\hat{u} \in H^{4}(\hat{K})$ associe la fonction d'interpolation $\hat{\Lambda}(\hat{a}) \hat{u}$ définie par des relations analogues aux relations (3.2)-(3.3). $\mathrm{n}^{\circ}$ août $1974, \mathrm{R}-2$. 
Il s'agit donc de montrer que

$$
\sup _{\hat{a} \in \hat{B}}\|\hat{\Lambda}(\hat{a})\|_{\mathfrak{L}_{\left(H^{4}(\hat{K}) ; H^{m}(\hat{K})\right)}=C(\hat{B})<+\infty .}
$$

Avec les notations de la figure 3 , la fonction $\hat{\Lambda}(\hat{a}) \hat{u}$ s'écrit

$$
\begin{aligned}
\hat{\Lambda}(\hat{a}) \hat{u} & =\sum_{i=1}^{3} \hat{u}\left(\hat{a}_{i}\right) \hat{p}_{i}(\hat{a}, .) \\
& +\sum_{i=1}^{3} \sum_{k=1}^{3}\left\langle D \hat{u}\left(\hat{a}_{i}\right),\left(\hat{a}_{k}-\hat{a}_{i}\right)\right\rangle \hat{q}_{i, k}(\hat{a}, .) \\
& +\sum_{i=1}^{3}\left\langle D \hat{u}\left(\hat{b}_{i}\right),\left(\hat{a}-\hat{b}_{i}\right)\right\rangle \hat{r}_{i}(\hat{a}, .),
\end{aligned}
$$

les fonctions $\hat{p}_{i}, \hat{q}_{i, k}$ et $\hat{r}_{i}$ formant une base de l'espace

$$
\hat{P}=\left\{\hat{p} \in \mathcal{C}^{1}(\hat{K}), \hat{p} \mid \hat{K}_{i} \in P_{3}, 1 \leqslant i \leqslant 3\right\}
$$

associé à l'élément $\hat{K}$.

Sur chacun des triangles $\hat{K}_{i}$, l'une quelconque des fonctions de base définies ci-dessus est un polynôme du troisième degré dont les coefficients sont obtenus par la résolution d'un système linéaire; or les coefficients de ce système linéaire dépendent continûment du point $\hat{a}$, puisque ce sont des polynômes en les coordonnées du point $\hat{a}$ : dans ces conditions, les coefficients des trois polynômes définissant une fonction de base sont des fonctions continues du point $\hat{a}$ et la majoration (3.6) résulte de cette continuité.

(iii) Il reste enfin à évaluer les quantités $|\Pi u-\Lambda u|_{m, K}$ pour $m=0,1,2$. Pour cela, nous utilisons une technique développée par P.-A. Raviart et l'auteur (cf. [6]) : Compte tenu des propriétés respectives d'interpolation des fonctions $\Pi u$ et $\Lambda u$, on peut écrire leur différence sous la forme

$$
\Pi u-\Lambda u=\sum_{i=1}^{3} \frac{\partial}{\partial n}(u-\Lambda u)\left(b_{i}\right)\left\langle n_{i},\left(a-b_{i}\right)\right\rangle s_{i},
$$

$n_{i}$ désignant le vecteur unitaire porté par la normale extérieure au point $b_{i}$, les polynômes $s_{i}$ étant des fonctions de base de l'espace $P$, associées à l'application d'interpolation $\Lambda$. L'évaluation de l'expression $\frac{\partial}{\partial n}(u-\Lambda u)\left(b_{i}\right)$ se fait alors par l'intermédiaire de la théorie affine; on obtient finalement

$$
|\Pi u-\Lambda u|_{m, K} \leqslant C h^{4-m}|u|_{4, K} \quad \text { pour } \quad m=0,1,2 .
$$

L'inégalité (3.1) est alors une conséquence des relations (3.4), (3.5), (3.6) et (3.7). 
REmaRQue. Utilisant la technique des formules de Taylor à plusieurs points développée dans [3], on démontrerait de façon analogue l'existence d'une constante $C$, indépendante des éléments d'une famille régulière, telle que

$$
\sup _{\substack{x \in K \\|\alpha|=m}}\left|\partial^{\alpha}(u-\Pi u)\right| \leqslant C h^{4-m} \sup _{\substack{x \in K \\|\alpha|=4}}\left|\partial^{\alpha} u\right| \quad \text { pour } \quad m=0,1 .
$$

\section{REFERENCES}

[1] Ciarlet P. G., Conforming and nonconforming finite element methods for solving the plate problem, à paraître dans les comptes rendus de la Conference on the Numerical Solution of Differential Equations, Université de Dundee, 3-6 juillet 1973.

[2] Ciarlet P. G., Quelques méthodes d'éléments finis pour le problème d'une plaque encastrée, à paraître dans les comptes rendus du Colloque International sur les Méthodes de Calcul Scientifique et Technique, I.R.I.A., Le Chesnay, 17-21 décembre 1973.

[3] Ciarlet P. G. et Raviart P.-A., General Lagrange and Hermite interpolation in $R^{n}$ with applications to finite element methods. Arch. Rational Mech. Anal., 46 (1972), 177-199.

[4] Ctarlet P. G. et Wagschal C., Multipoint Taylor formulas and applications to the finite element method, Numer. Math., 17, (1971), 84-100.

[5] Clough R. W. et Tocher J. L., Finite element stiffness matrices for analysis of plates in bending, Conference on Matrix Methods in Structural Mechanics, Wright Patterson A.F.B., Ohio, 1965.

[6] Raviart P.-A., Méthode des Eléments Finis, Université de Paris VI, Paris, 1972.

[7] ZIENkIEwicz O. C., The Finite Element Method in Engineering Science, McGrawHill, London, 1971. 